\title{
Ultra High Energy Cosmic Rays and Inflation Relics.
}

\author{
Vadim A. Kuzmin ${ }^{a}$ and Igor I. Tkachev ${ }^{a, b}$ \\ ${ }^{a}$ Institute for Nuclear Research, Russian Academy of Sciences, \\ 60th October Anniversary Prosp. 7a, Moscow 117312, Russia and \\ ${ }^{b}$ TH Division, CERN, CH-1211 Geneva 23, Switzerland
}

\begin{abstract}
There are two processes of matter creation after inflation that may be relevant to the resolution of the puzzle of cosmic rays observed with energies beyond GZK cut-off: 1) gravitational creation of superheavy (quasi)stable particles, and 2) nonthermal phase transitions leading to formation of topological defects. We review both possibilities.
\end{abstract}

Key words: Cosmic rays; Dark matter; Early universe

\section{Introduction}

Cosmological and astrophysical considerations are able to provide the strongest restrictions on parameters of particle physics models and even rule out some classes of models entirely. This is especially valuable when the model is unrestricted by laboratory experiments (which is often the case). Among famous results which made a strong impact on model building is the cosmological domain wall problem which appears in models with spontaneous breaking of discrete symmetries [1] and the problem of magnetic monopoles in Grand Unified theories [2]. In return, studies of cosmological phase transitions [3] and of the dynamics of bubbles of a metastable vacuum [4] lead to the change of basic concepts of the cosmology of the early Universe, and inflationary cosmology [5-8] was born (for reviews see $[9,10]$ ). Inflation gives a possible solution to horizon, flatness and homogeneity problems of "classical" cosmology [6]. Inflation was designed to solve the problem of unwanted relics, like magnetic monopoles. It was promptly realized [11] that inflation can generate small amplitude large scale density fluctuations which are the necessary seeds 
for the galaxy and the large scale structure formation in the Universe. This elevates inflation from the rank of a "broad brush problem solver" into the rank of a testable hypotheses. And testable in fine details, as rapidly accumulating data on cosmic microwave background fluctuations (starting from COBE detection [12] through numerous balloon and ground based CMBR experiments and with culmination at MAP [13] and PLANK [14] anticipated detailed maps of anisotropy of the microwave sky) and huge galaxy catalogs like the already collecting data SLOAN digital sky surview [15] will provide a wealth of cosmological information.

Inflation is generally assumed to be driven by the special scalar field $\phi$ known as the inflaton. During inflation, the inflaton field slowly rolls down towards the minimum of its potential. Inflation ends when the potential energy associated with the inflaton field becomes smaller than the kinetic energy, which happens when magnitude of the inflaton field decreases below the Plank scale, $\phi \lesssim$ $M_{\mathrm{Pl}}$ and "cold" coherent oscillations of the inflaton field commence. These oscillations contained all the energy of the Universe at that time.

All matter in the Universe was created in reheating, which is nothing but decay of the zero momentum mode of inflation oscillations. The process is obviously of such vital importance that here too one may hope to find some observable consequences, specific for the process itself and for particular models of particle physics, despite the fact that scales relevant for the reheating are very small. And, indeed, we now believe that there can be some clues left. Among those are: topological defects production in non-thermal phase transitions [16], GUT scale baryogenesis [17], generation of primordial background of stochastic gravitational waves at high frequencies [18], just to mention a few. However, the most interesting can be a possible relation to a mounting puzzle of the Ultra High Energy Cosmic Rays (UHECR) [19].

When a proton (or neutron) propagates in CMB, it gradually looses energy colliding with photons and creating pions. There is a threshold energy for the process, so it is effective for very energetic nucleons only, which leads to the Greisen-Zatsepin-Kuzmin (GZK) cutoff [20] of the high energy tail of the spectrum of cosmic rays. All this means that detection of, say, $3 \times 10^{20} \mathrm{eV}$ proton would require its source to be within $\sim 50 \mathrm{Mpc}$. However, several well established events above the cut-off were observed by Yakutsk [21], Haverah Park [22], Fly Eye [23] and AGASA [24] collaborations (for the recent reviews see Refs. [25,26]).

Results from the AGASA experiment [27] are shown in Fig. 1. The dashed curve represents the expected spectrum if conventional extragalactic sources of UHECR would be distributed uniformly in the Universe. This curve displays the theoretical GZK cut-off, but one observes events which are way above it. (Numbers attached to the data points show the number of events observed in 


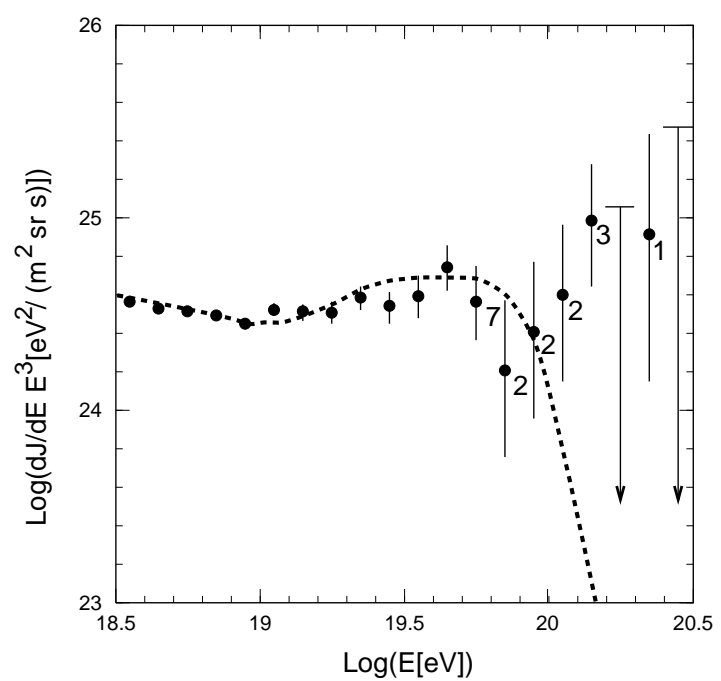

Fig. 1. AGASA data set [27], February 1990 - October 1997.

each energy bin.) Note that no candidate astrophysical source, like powerful active galaxy nuclei, were found in the directions of all six events with $E>10^{20}$ $\mathrm{eV}[27]$ (at these energies cosmic rays experience little deflection by galactic magnetic fields).

Is some unexpected astrophysics at work here or this is at last an indication of the long awaited new physics?

There are two logical possibilities to produce UHE cosmic rays: either charged particles have to be accelerated to energies $E>10^{20} \mathrm{eV}$, or UHECR originate in decays of heavy X-particles, $m_{X}>10^{12} \mathrm{GeV}$. Maximum energy which can be achieved in an accelerating site of the size $R$ which has the magnetic field strength $B$ is [28]:

$$
E<10^{20} Z \frac{B}{\mu \mathrm{G}} \frac{R}{\mathrm{Mpc}} \mathrm{eV} .
$$

A magnetic field is required either to keep the particle confined within the accelerating region or to produce an accelerating electric field. For protons $(Z=1)$ a few sources satisfy this condition: pulsars, active galactic nuclei (AGNs) and radio-galaxies. However, energy losses (pair production and meson photoproduction) restrict the maxim energy to $E<10^{16} \mathrm{eV}$ in pulsars and AGNs $[25,29]$, while radio-galaxies that lie along the arrival directions of UHECR are situated at large cosmological distances, $\gtrsim 100 \mathrm{Mpc}[30]$, i.e. beyond the GZK radius. Similar conclusion seem to be true with respect to cosmological Gamma Ray Bursts as a possible source of UHECRs [31].

New astrophysics which may work is a possibility to generate UHECR within 
GZK sphere in remnants of dead quasars [32] (these are dormant galaxies which harbour supermassive spinning black hole).

New physics suggested as an explanation of UHE cosmic rays, range up to the violation of the Lorentz invariance [33]. Among less radical extensions of the standard model are:

- The existence of a particle which is immune to CMB in comparison with nucleons. In this scenario the primary particle is produced in remote astrophysical accelerators (e.g. radio-galaxies) and is able to travel larger cosmological distances while having energies above the GZK cut-off. There are variations to this scheme.

- Suprsymmetric partner of gluon, the gluino, can form bound states with quarks and gluons. If gluino is light and quasistable (see e.g. [34,35]), the lightest gluino containing baryons will have sufficiently large GZK threshold to be such a messenger [34] and as a hadron it will be able to produce normal air showers in the Earth's atmosphere. However, there are strong arguments due to Voloshin and Okun [36], against light quasistable gluino based on constraints on the abundance of anomalous heavy isotopes which also will be formed as bound states with gluino.

- High energy (anti)neutrinos produced in distant astrophysical sources will annihilate via $Z^{0}$ resonance on the relic neutrinos and produce energetic gamma or nucleon [37]. The relic neutrino masses in the eV range are consistent with this scenario [38], as well as with the Super-Kamiokande results. The required high density of the relic neutrinos is achieved if gravitational clumping takes place [37] or if the Universe has a significant lepton asymmetry in background neutrinos [38]. Even then the total luminosity of the neutrino sources in the Universe must be as high as $10^{-2}-10^{1}$ of its photon luminosity, and, therefore, neutrino-only sources are called for by the upper bound from the flux of the cosmic rays [39]. An independent constraint on the density of the relic neutrinos comes from CMBR and already the present data start to be challenging for models with large neutrino asymmetry [40].

- Another class of suggestions is related to topological defects. UHECR are produced when topological defects decompose to constituent fields (X-particles) which in turn decay [41]. Maximum energy is not a problem here, but in models which involve string [42] or superconducting string [41] networks, the typical separation between defects is of order of the Hubble distance and thus these models are subject to GZK cut-off. Models in which defects can decay "locally" include networks of monopoles connected by strings (necklaces) [43], vortons (charge and current carrying loops of superconducting strings stabilized by angular momentum) [44], and monopolonium (bound monopole-antimonopole pairs) [45]. Finally, magnetic monopoles accelerated by intergalactic magnetic fields were also considered as primary UHECR particls [46]. 


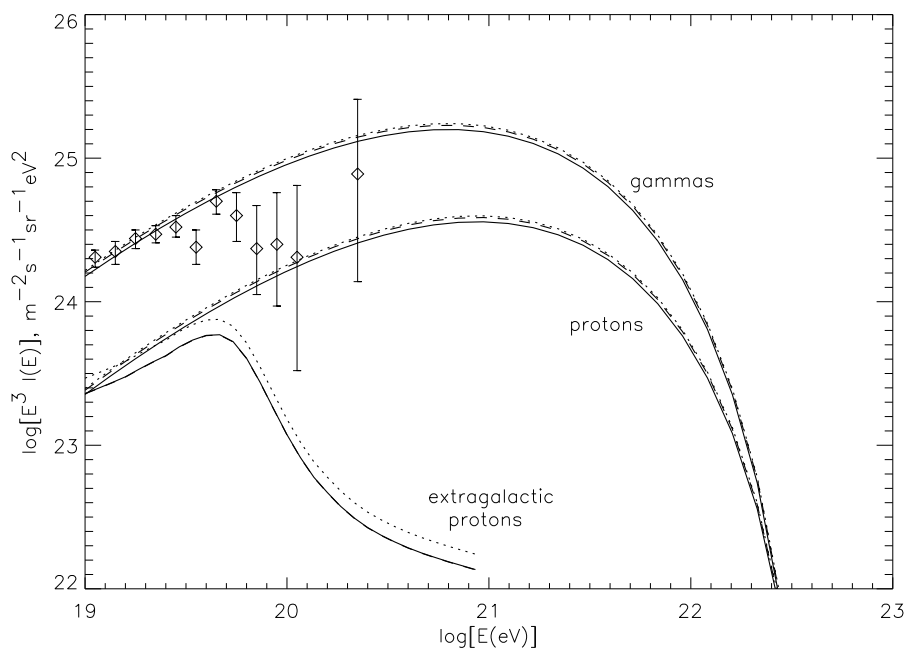

Fig. 2. Predicted fluxes from decaying X-particles, as calculated in Ref. [49] and the data. Latest AGASA results, Fig. 1, are not shown.

- Conceptually the simplest possibility is that UHECR are produced cosmologically locally in decays of some new particle [47,48]. GZK cut-off is automatically avoided but the candidate $X$-particle must obviously obey constraints on mass, number density and lifetime.

\section{UHECR from decaying particles}

In order to produce cosmic rays in the energy range $E>10^{11} \mathrm{GeV}$, the decaying primary particle has to be heavy, with the mass well above GZK cutoff, $m_{X}>10^{12} \mathrm{GeV}$. The lifetime, $\tau_{X}$, cannot be much smaller than the age of the Universe, $t_{U} \approx 10^{10} \mathrm{yr}$. Given this shortest possible lifetime, the observed flux of UHE cosmic rays will be generated with the rather low number density of $X$-particles, $\Omega_{X} \sim 10^{-12}$, where $\Omega_{X} \equiv m_{X} n_{X} / \rho_{\text {crit }}, n_{X}$ is the number density of X-particles and $\rho_{\text {crit }}$ is the critical density. On the other hand, $\mathrm{X}$-particles must not overclose the Universe, $\Omega_{X}<1$. With $\Omega_{X} \sim 1$, the Xparticles may play the role of cold dark matter and the observed flux of UHE cosmic rays can be matched if $\tau_{X} \sim 10^{22}$ yr.

Spectra of UHE cosmic rays arising in decays of relic X-particles were successfully fitted to the data for $m_{X}$ in the range $10^{12}<m_{X} / \mathrm{GeV}<10^{14}[49,50]$. For example, the fit of Berezinskii et. al. [49] to observed fluxes of UHECR assuming $m_{X} \approx 10^{14} \mathrm{GeV}$ is shown in Fig. 2. Beside the mass of the X-particle there is another parameter which controls the flux of the cosmic rays from decaying particles: namely, the ratio of X-particles number density and their lifetime. For the fit in Fig. 2 it was used $\left(\Omega_{X} / \Omega_{\mathrm{CDM}}\right)\left(t_{U} / \tau_{X}\right)=5 \times 10^{-11}$. 
The problem of the particle physics mechanism responsible for a long but finite lifetime of very heavy particles can be solved in several ways. For example, some otherwise conserved quantum number carried by X-particles may be broken very weakly due to instanton transitions [47], or quantum gravity (wormhole) effects [48]. If instantons are responsible for $X$-particle decays, the lifetime is estimated as $\tau_{X} \sim m_{X}^{-1} \cdot \exp \left(4 \pi / \alpha_{X}\right)$, where $\alpha_{X}$ is the coupling constant of the relevant gauge interaction. The lifetime will fit the allowed window if the coupling constant (at the scale $m_{X}$ ) is $\alpha_{X} \approx 0.1$ [47].

A class of natural candidates for superheavy long-living particles which arise in string and M theory was re-evaluated recently in Refs. [51] and particles with desired mass and long life-time were identified. Other interesting candidates were found among adjoint messengers in gauge mediated supergravity models [52] and in models of superheavy dark matter with discrete gauge symmetries [53]. Superheavy dark matter candidates in superstrings and supregarvity models were considered also in Refs. [54].

Below we address the issue of X-particle abundance.

\section{Superheavy particle genesis in the early Universe}

Seperheavy particles can be created in the early Universe by several mechanisms. Among those are:

- Non-equilibrium "thermal" production in scattering or decay processes in primordial plasma [47,48].

- Production during decay of inflaton oscillations ("preheating") [55-58].

- Direct gravitational production from vacuum fluctuations during inflation $[59,19,60]$.

In any case the final ratio of the density in $X$ particles to the entropy density is normalized by the reheating temperature. The reheating temperature is limited to the value below $10^{8}-10^{9} \mathrm{GeV}$ in supergravity models with decaying heavy gravitino [61]. This restricts model parameters when "thermal" mechanism of heavy particle production is operative (but does not rule it out $[47,48,62])$.

The last two mechanisms are closely related to each other and both can be described on equal footing within frameworks of a single unified approach: particle creation in external time varying background. However, while the outcome of the second mechanism is highly dependent upon the strength of the interaction of the X-field to the inflaton, no coupling (e.g. to the inflaton or plasma) is needed in the third mechanism when the temporal change of 
the metric is the single cause of particle production. Even absolutely sterile particles are produced by the third mechanism which may be relevant for very long-living superheavy particles. Resulting abundance is quite independent of the detailed nature of the particle which makes the superheavy (quasi)stable $\mathrm{X}$-particle a very interesting dark matter candidate.

We concentrate here on the second and third mechanisms and from the start we introduce coupling of the X-field to the inflaton for uniformity of discussion. The limit of zero coupling will correspond to pure gravitational production.

In the case of a heavy scalar field $X$ we consider the model

$$
L=\frac{1}{2}\left(\partial_{\mu} \phi\right)^{2}-V(\phi)+\frac{1}{2}\left(\partial_{\mu} X\right)^{2}-\frac{1}{2}\left(M_{X}^{2}-\xi R\right) X^{2}-\frac{g^{2}}{2} \phi^{2} X^{2}
$$

Here $V(\phi)$ is the inflaton potential. In simple "chaotic" [8] models of inflation the inflaton is either "massive" with the scalar potential $V(\phi)=m_{\phi}^{2} \phi^{2} / 2$, or "massless", $V(\phi)=\lambda \phi^{4} / 4$. Normalization to the large scale structure requires $m_{\phi}^{2} / M_{\mathrm{Pl}}^{2} \approx 10^{-12}$ in the former model and $\lambda \approx 10^{-13}$ in the latter model. The constant $\xi$ describes direct coupling to the space-time curvature $R$, with $\xi=0$ corresponding to the minimal coupling and $\xi=1 / 6$ being the case of conformal coupling.

Fermion field ( $\operatorname{spin} \frac{1}{2}$ ) is conformally coupled to gravity. In addition to standard kinetic and mass terms it also may have coupling to the inflaton, $V_{\frac{1}{2}}=$ $g \phi \bar{X} X$

It is convenient to work in conformal metric $d s^{2}=a(\eta)^{2}\left(d \eta^{2}-d \mathbf{x}^{2}\right)$ with rescaled fields, $\varphi \equiv \phi a(\eta)$ and $\chi \equiv X a(\eta)^{s}$, where $s=1$ and $s=\frac{3}{2}$ for scalar and fermion fields respectively. In what follows we measure time and space intervals in units of inflaton mass, $\tau \equiv m \eta$.

\subsection{Quantum fields in classical backgrounds}

Here we summarise the basic formalism of particle creation in external classical background (e.g. space-time metric of an expanding universe or oscillating inflaton field). For more details see e.g. Refs. [63-66].

i) Spin 0 bosons.

A real scalar field is Fourier expanded in a comoving box

$$
\chi(\tau, \mathbf{x})=\sum_{\mathbf{k}}\left[\chi_{k}(\tau) a_{\mathbf{k}}+\chi_{k}^{*}(\tau) a_{-\mathbf{k}}^{\dagger}\right] e^{i \mathbf{k x}} .
$$


Annihilation and creation operators commute except for $\left[a_{\mathbf{k}}, a_{\mathbf{k}}^{\dagger}\right]=1$. The mode functions, $\chi_{k} \equiv \chi_{k}(\tau)$ of a scalar Bose field are solutions of the oscillator equation

$$
\chi_{k}^{\prime \prime}+\omega_{k}^{2}(\tau) \chi_{k}=0
$$

with the time dependent frequency

$$
\omega_{k}^{2}(\tau)=k^{2}+\frac{a^{\prime \prime}}{a}(6 \xi-1)+m_{\chi}^{2} a^{2}+4 q \varphi^{2}
$$

where $^{\prime} \equiv d / d \tau$ and

$$
m_{\chi}^{2} \equiv \frac{M_{X}^{2}}{m^{2}}, \quad q \equiv \frac{g^{2} \phi^{2}(0)}{4 m^{2}}
$$

Here $\phi(0)$ is the value of the inflaton field when it starts to oscillate (which corresponds to normalization $\varphi(0)=1$ ).

Let $\omega_{k}$ at some time interval satisfy the adiabatic condition $\left|\omega_{k}^{\prime}\right| / \omega_{k}^{2} \ll 1$. We can choose solutions of Eq. (4) which enter decomposition Eq. (3) to be positive-frequency modes $\chi_{k}=\omega_{k}^{-1 / 2} e^{-i \omega_{k} \tau}$ and define vacuum state $a_{\mathbf{k}}|0\rangle=0$. The number of particles in a non-vacuum state will be constant during evolution through this adiabatic interval. Let the adiabatic condition be violated for some time and then the system enters another adiabatic interval. In that interval we can define another set of positive-frequency modes and corresponding vacuum. Initial positive-frequency modes evolved through non-adiabatic region will not coincide with "out" state modes, but one set of modes can be expressed in terms of the other. This decomposition is called Bogolyubov transformation [67]. Since one and the same field is expanded with the use of two different sets of mode functions, the Fourier coefficients are also related to each other

$$
a_{\mathbf{k}}^{\text {out }}=\alpha_{\mathbf{k}} a_{\mathbf{k}}+\beta_{\mathbf{k}}^{*} a_{\mathbf{k}}^{\dagger}
$$

It follows immediately that the initial vacuum state at late times contains particles

$$
\left\langle 0\left|a_{\mathbf{k}}^{\dagger \text { out }} a_{\mathbf{k}}^{\text {out }}\right| 0\right\rangle=\left|\beta_{k}\right|^{2}
$$

Technically it is easier to find Bogolyubov's coefficients by diagonalizing Hamiltonian of the field $X$. For any time moment $\tau$ this procedure gives

$$
\left|\beta_{k}\right|^{2}=\frac{\left|\chi_{k}^{\prime}\right|^{2}+\omega^{2}\left|\chi_{k}\right|^{2}-2 \omega}{4 \omega}
$$


where mode functions are solutions of Eq. (4) with initial (vacuum) conditions

$$
\chi_{k}(0)=\omega^{-1 / 2}, \quad \chi_{k}^{\prime}(0)=-i \omega \chi_{k} .
$$

ii) Spin 1/2 fermions.

The relevant mode functions of the Fermi field satisfy the oscillator equation with the complex frequency

$$
\chi_{k}^{\prime \prime}+\left(\omega_{k}^{2}-i m_{\mathrm{eff}}^{\prime}\right) \chi_{k}=0
$$

where the real part of the frequency is given by $\omega_{k}^{2}=k^{2}+m_{\mathrm{eff}}^{2}$ and $m_{\mathrm{eff}}=$ $m_{\chi} a+\sqrt{q} \varphi$. We choose

$$
\chi_{k}(0)=\sqrt{1-\frac{m_{\mathrm{eff}}}{\omega}}, \quad \chi_{k}^{\prime}(0)=-i \omega \chi_{k}
$$

as the initial conditions. In this case we find per spin state

$$
\left|\beta_{k}\right|^{2}=\frac{\omega-m_{\mathrm{eff}}-\operatorname{Im}\left(\chi_{k} \chi_{k}^{*^{\prime}}\right)}{2 \omega} .
$$

Finally, the number density of $X$-particles created by time varying background is

$$
n_{X}=\frac{1}{2 \pi^{2} a^{3}} \sum_{s} \int\left|\beta_{k}\right|^{2} k^{2} d k
$$

where $\sum_{s}$ is the sum over spin states. The expression (14) gives the number density of particles only, with an equal amount of antiparticles being created in the case of charged fields.

\subsection{Gravitational creation of particles}

It was noticed $[59,19]$ that superheavy particles are produced gravitationally in the early Universe from vacuum fluctuations and their abundance can be correct naturally, if the standard Friedmann epoch in the Universe evolution was preceded by the inflationary stage. This is a fundamental process of particle creation unavoidable in the time varying background and it requires no interactions. Temporal change of the metric is the single cause of particle production. Basically, it is the same process which during inflation had generated primordial large scale density perturbations. No coupling (e.g. to the inflaton 
or plasma) is needed. All one needs are stable (very long-living) X-particles with mass of order of the inflaton mass, $m_{X} \approx 10^{13} \mathrm{GeV}$. Inflationary stage is not required to produce superheavy particles from the vacuum. Rather, the inflation provides a cut off in excessive gravitational production of heavy particles which would happen in the Friedmann Universe if it would start from the initial singularity [19]. Resulting abundance is quite independent of the detailed nature of the particle which makes the superheavy (quasi)stable $\mathrm{X}$-particle a very interesting dark matter candidate. So, we start our consideration with gravitational creation of particles, i.e. we put $g=0$ (or equivalently $q=0)$ in the formulas above.

\subsubsection{Friedmann Cosmology}

For particles with conformal coupling to gravity (fermions or scalars with $\xi=1 / 6)$, it is the particle mass which couples the system to the background expansion and serves as the source of particle creation. Therefore, just on dimensional grounds, we expect

$$
n_{X} \propto m_{X}^{3} a^{-3}
$$

at late times when particle creation diminishes. In Friedmann cosmology, $a \propto$ $(m t)^{\alpha} \propto(m / H)^{\alpha}\left(\alpha=\frac{1}{2}\right.$ and $\alpha=\frac{3}{2}$ for radiation and matter dominated expansion respectively). We conclude that the anticipated formulae for the $\mathrm{X}$-particles abundance can be parameterised as

$$
n_{X}=C_{\alpha} m_{X}^{3}\left(\frac{H}{m_{X}}\right)^{3 \alpha} .
$$

On the other hand, it is expansion of the Universe which is responsible for particle creation. Therefore, this equation which describes simple dilution of already created particles, is valid when expansion becomes negligible, $H \ll$ $m_{X}$. This means also that particles with $m_{X} \gg H$ cannot be created by this mechanism. Creation occurs when $H \sim m_{X}$. The coefficient $C_{\alpha}$ depends upon the background cosmology only, and it can be found numerically [19], see Fig. 3.

In particular, for the radiation dominated Universe, which was studied also in Ref. [63], one finds $\Omega_{X} \equiv \rho_{X} / \rho_{c}=m_{X} n_{X} 32 \pi G t^{2} / 3$ with the present value of $\Omega_{X}$ being equal to $\Omega_{X} \sim 2 \times 10^{-2}\left(m_{X}^{2} / M_{\mathrm{Pl}}^{2}\right) \sqrt{m_{X} t_{e}}$, where $t_{e}$ is the time of equal densities of radiation and matter in the $\Omega=1$ Universe. This gives $\Omega_{X} \sim\left(m_{X} / 10^{9} \mathrm{GeV}\right)^{5 / 2}$. Stable weakly interacting particles with $m_{X} \gtrsim 10^{9}$ $\mathrm{GeV}$ will overclose the Universe even if initially they were in a vacuum state and were created from the vacuum during the regular radiation dominated stage of the Universe evolution. 


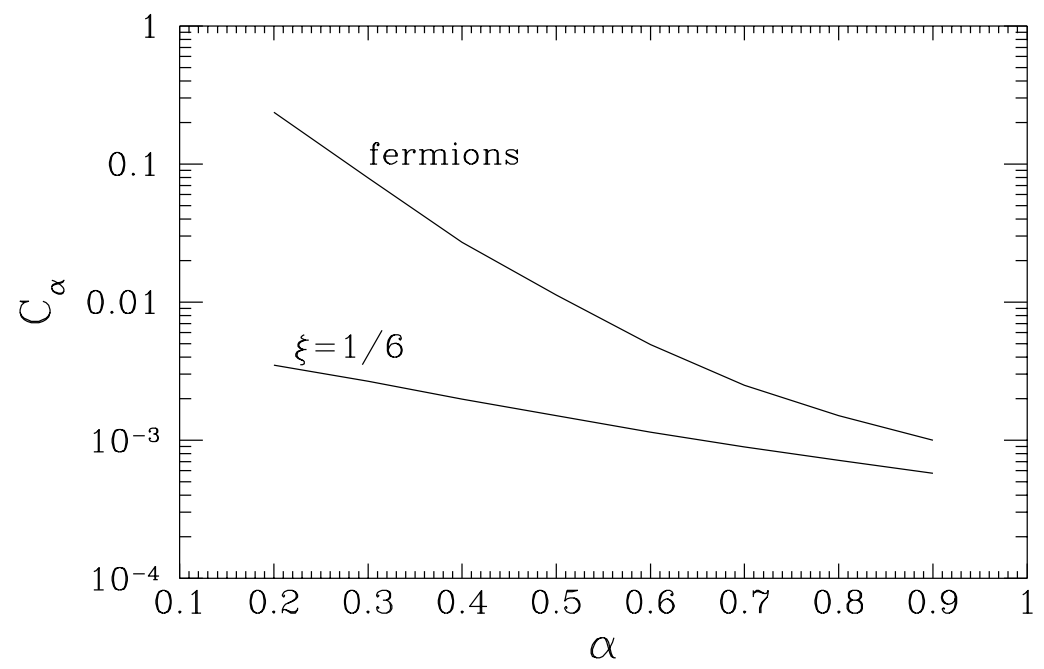

Fig. 3. The coefficient $C_{\alpha}$, defined in Eq. (16), is shown as a function of $\alpha$ for the background cosmology with a power law scale factor $a \propto t^{\alpha}$, Ref. [60].

There is no room for superheavy particles in our Universe if it started from the initial Friedmann singularity [19], since the value of the Hubble constant is limited from above only by the Planck constant in this case.

\subsubsection{Inflationary Cosmology}

However, this restriction will not be valid if inflation separates initial conditions, whatever they were, from the observable Universe. In inflationary cosmology the Hubble constant (in effect) did not exceeded the inflaton mass, $H<m_{\phi}$. The mass of the inflaton field has to be $m_{\phi} \sim 10^{13} \mathrm{GeV}$ as constrained by the amplitude of primordial density fluctuations relevant for the large scale structure formation. Therefore, direct gravitational production of particles with $m_{X}>H \sim 10^{13} \mathrm{GeV}$ has to be suppressed in inflationary cosmology.

Particle creation from vacuum fluctuations during inflation (or in the de Sitter space) was extensively studied $[68,69]$, usually in the case of small $m_{X}$ and in application to generation of density fluctuations necessary for the large scale structure formation. The characteristic quantity which is usually cited in this applications, the variance of the field, $\left\langle X^{2}\right\rangle$, is defined by an expression similar to Eq. (14), in the typical case $\alpha_{k} \approx-\beta_{k}$ the integrand is being multiplied by the factor $2 \sin ^{2}\left(\omega_{k} \tau\right) / \omega_{k}$. For example, for the scalar Bose field with the minimal coupling to the curvature, $\left\langle X^{2}\right\rangle=3 H_{i}^{4} / 8 \pi^{2} m_{X}^{2}$ if $m_{X} \ll H_{i}[68,69]$. For massless self-interacting field $\left\langle X^{2}\right\rangle \approx 0.132 H_{i}^{2} / \sqrt{\lambda}$ [70]. Particle creation for the specific case of the Hubble dependent effective mass, $m_{X}(t) \propto H(t)$, was considered in Ref. [71]. 


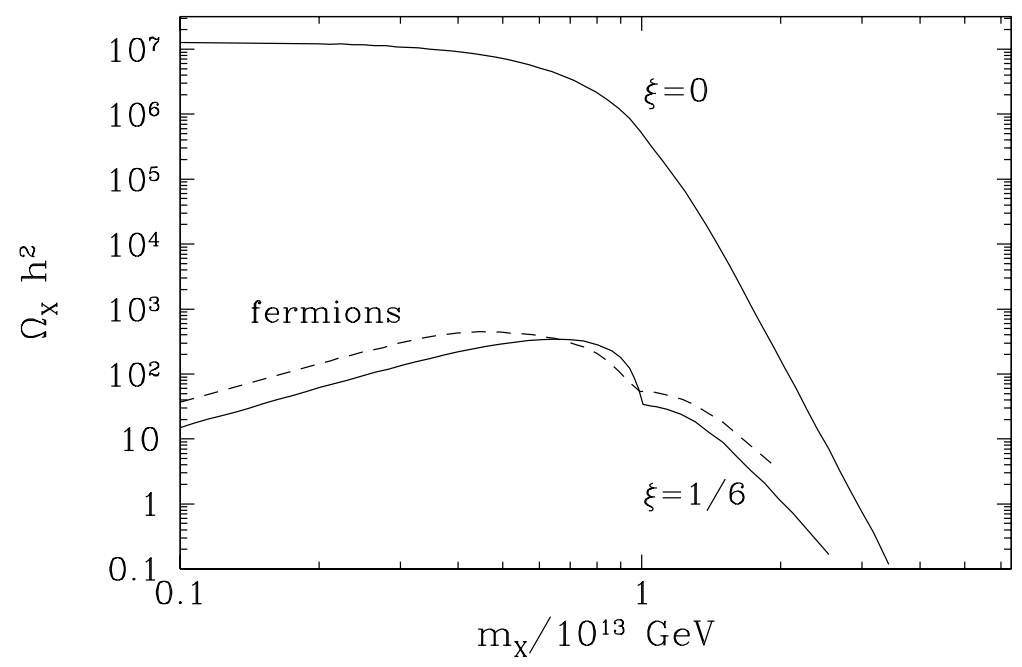

Fig. 4. Ratio of the energy density in $X$-particles, gravitationally generated in inflationary cosmology, to the critical energy density is shown as a function of X-particle mass, Ref. [60].

Results [60] of direct numerical integration of gravitational creation of superheavy particles in chaotic inflation model with the potential $V(\phi)=m_{\phi}^{2} \phi^{2} / 2$ is shown in Fig. 2.

This figure was calculated assuming $T_{\mathrm{R}}=10^{9} \mathrm{GeV}$ for the reheating temperature. (At reheating the entropy of the Universe was created in addition to $\mathrm{X}$-particles. In general, multiply this figure by the ratio $T_{\mathrm{R}} / 10^{9} \mathrm{GeV}$ and devide it by the fractional entropy increase per comoving volume if it was significant at some late epoch.) The reheating temperature is constrained, $T_{\mathrm{R}}<10^{9} \mathrm{GeV}$, in supergravity theory [61]. We find that $\Omega_{X} h^{2}<1$ if $m_{X} \approx($ few $) \times 10^{13} \mathrm{GeV}$. This value of mass is in the range suitable for the explanation of UHECR events [19]. Gravitationally created superheavy X-particles can even be the dominating form of matter in the Universe today if $\mathrm{X}$-particles are in this mass range $[59,19]$.

\subsubsection{Isocurvature fluctuations in superheavy particle matter}

In numerical calculations, Ref. [60], it was found that the variance $\left\langle X^{2}\right\rangle$ of the field $X$ measured at the end of inflation is independent upon $m_{X}$ if the mass of $X$ is small and coupling to curvature is minimal. At some later epoch when $H \approx m_{X}$ (which will be long after the end of inflation if $\mathrm{X}$ is a light field) the field $X$ starts to oscillate on all scales, including $k=0$. Only at this time, which we denote by $t_{X}$, all field fluctuations are transformed into the non-zero particle density and we can use $\rho_{X}=m_{X} n_{X} \approx m_{X}^{2}\left\langle X^{2}\right\rangle$. The variance of $X$ fluctuations was unchanged on large scales, starting from the end of inflation 


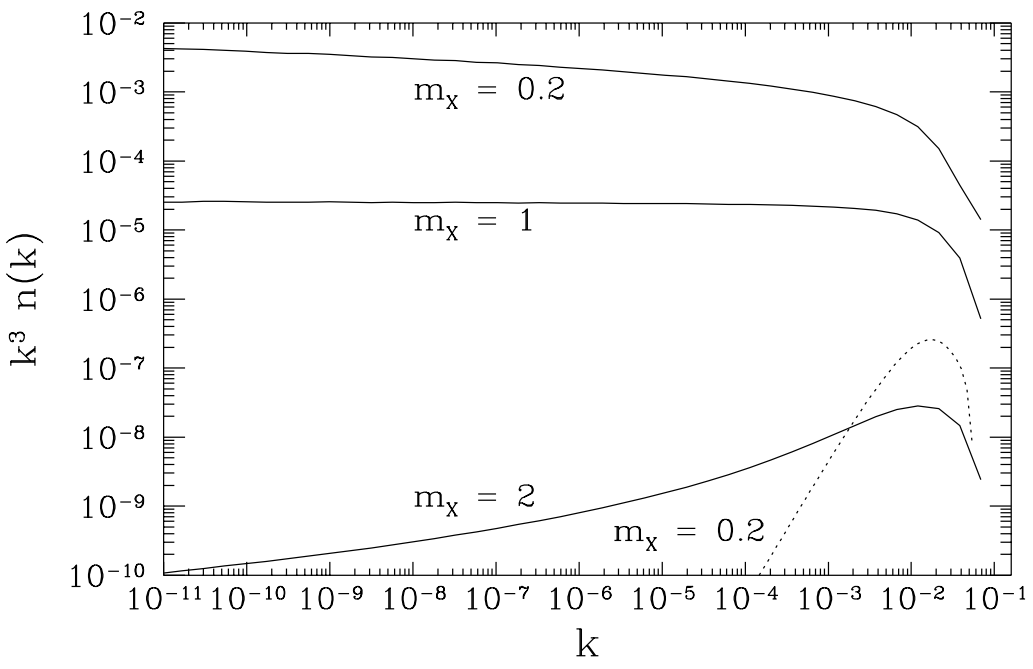

Fig. 5. Spectrum of created particles, $k^{3} n(k)$, in a model with massive inflaton is shown for several choices of the mass of scalar X-particle with the minimal coupling (solid lines) and the conformal coupling (dotted line), Ref. [60]. Masses and momenta, $k$, are given in units of the inflaton mass.

down to the time $t_{X}$. So, when the field starts to oscillate $\rho_{X} \propto m_{X}^{2}$. However, the energy density of the inflaton field, $\rho=3 H^{2} / 8 \pi G$, decreased during this time interval in proportion to $H^{2}\left(t_{X}\right) / H^{2}(0) \approx m_{X}^{2} / H^{2}(0)$. That is why the ratio of the energy density in $X$-particles to the total energy density does not depend on $m_{X}$ when measured at $t>t_{X}$, see Fig. 4 .

Variance of the field $\mathrm{X}$ is different from the usually calculated for the fixed de Sitter inflationary background because we consider the actual evolution of the scale factor and the value of the Hubble parameter is not constant during inflation, being larger at earlier times. Correspondingly, the number of created particles per decade of $k$ grows logarithmically towards small $\mathrm{k}$ if $m_{X}$ is small. (Power spectrum behaves similarly.) The examples of the particle number, $k^{3} n_{X}(k)$, for several values of $m_{X}$ are shown in Fig. 5 at the moment corresponding to 10 completed inflaton oscillations. The particle momentum is measured in units of the inflaton mass. In contrast to this, in the fixed de Sitter background $4 \pi^{2}\left\langle X^{2}\right\rangle \approx H^{2} \int d \ln k(k / H)^{3-2 \nu}$ with $(3-2 \nu) \approx 2 m_{X}^{2} / 3 H^{2}$ at small $m_{X}$, and consequently $\left\langle X^{2}\right\rangle \propto 1 / m_{X}^{2}$. Note that the power spectrum in the fixed de Sitter background grows towards large values of $k$, which is opposite to the behaviour of Fig. 5 .

Therefore, calculations which would be based on the customary procedure of matching a fixed de Sitter background to a subsequent Friedmann stage would give wrong results, with $\Omega_{X} \rightarrow \infty$ at $m_{X} \rightarrow 0$.

Matching is also dangerous in the case of large $m_{X}$. When the change is too abrupt, it generates artificial particles. This may easily happen for $m_{X}>m_{\phi}$, 
see e.g. [72] where excessive production was found. At $m_{X} \gtrsim m_{\phi}$ the number of created particles decreases exponentially with $m_{X}$.

As Fig. 5 shows, the power spectrum of fluctuations in X-particles is almost scale independent at small $\mathrm{k}$ if $m_{X} / m_{\phi} \approx 1$. Therefore, if such particles constitute a considerable fraction of dark matter, these fluctuations will be transformed into isocurvature density perturbations at late times and can affect large scale structure formation. Isocurvature fluctuations produce 6 times larger angular temperature fluctuations in cosmic microwave background radiation (CMBR) for the same amplitude of long-wavelength density perturbations compared to the adiabatic case [73]. Therefore, to fit observations by a single spectrum, the mass fluctuation spectrum in isocurvature cold dark matter cosmology must be tilted (with respect to scale invariant spectrum) to favor smaller scales.

Let the power spectrum of the field fluctuations be $k^{3} P_{X}(k) \propto k^{\beta}$. Fit to the second moments of the large-scale mass and cosmic microwave background distributions requires $\beta \gtrsim 0.25$. Models with $\beta$ ranging from 0.3 to 0.6 were considered in Refs. [74,75]. It is interesting that the spectrum is indeed correctly tilted for $m_{X}$ which is somewhat larger than the inflaton mass, see Fig. 5. On the other hand we see that lighter particles, $m_{X}<m_{\phi}$, with minimal coupling to gravity and $\Omega_{X} \approx 1$ are excluded.

\subsection{Preheating}

Considering gravitational creation of particles only, we would be left today with an oscillating inflaton field dominating the Universe. $\square$ To reheat the Universe we must couple the inflaton to some other fields. In oscillating background and in flat space-time the number density of particles grows exponentially: parametric resonance is always effective, see e.g. Ref. [66]. This would lead to explosive decay of oscillations. However, in an expanding universe the resonance is blocked by the redshift, which removes created particles out of the resonance bands. Thus, coherent oscillations of e.g. axion [77], or moduli fields [78], do not decay via parametric resonance in the expanding Universe. It is interesting that the field trapped in a (self-) gravitational well can burst in radiation, in principle $[79,80]$, but limitations on the relation between density and size of the clump make it hard to achieve critical conditions when elaborated dark matter models, like the axion [79,81] are considered (axion miniclusters are promising objects in this respect though [82]).

1 Exclusions from this rule exist if inflaton potential does not have a minimum and energy density in the inflaton field after inflation decreases faster than in conventional matter [76]. 
Narrow width of the resonance band is a prime reason of stability and such fields whill decay only when the Hubble constant $H$ becomes smaller than the particle width $\Gamma$ (i.e. the life-time of individual particle becomes smaller than the age of the Universe). This results in reheating temperature $T_{\mathrm{RH}} \sim \sqrt{\Gamma M_{\mathrm{Pl}}}$. For a long time it was believed [83] that this is the end of the story for the inflaton field, until it was realized [55] that the resonance parameter $q$, Eq. (6) can naturally be extremely large in the inflaton case ${ }^{2}$. Indeed, the coupling constant is multiplied by the ratio of initial amplitude of the field to its mass, squared, which for the inflation is $\sim 10^{10}$. The resonance is broad and it is impossible to red-shift the system out of the resonance. Resulting explosive decay is not actually a parametric resonance, since resonance parameter can change by orders of magnitude just during one period of oscillation $[56,85]$, but decay occurs anyway because each period of oscillations the adiabaticity conditions are violated during some short time intervals [56,85]. It is possible that the inflaton oscillations decay after only a dozen of oscillations.

Let us now consider this process and introduce a non-zero coupling of the inflaton and the $X$ fields. For the sufficiently large value of $q$ in Eq. (6) the gravitational production of particles is negligible. If the amount of produced particles is relatively small, the process of their creation still can be considered as creation by external time varying background (oscillating zero mode of the inflaton). However, production can be very efficient at large $q$ and back reaction of produced particles on the motion of the inflaton field has to be included. This restores conservation laws and, as a matter of fact, we are considering the decay of the inflaton into $X$ now. Here we restrict ourselves to the case of scalar X-particles.

The equation of motion for the inflaton field is

$$
\varphi^{\prime \prime}-\nabla^{2} \varphi-\frac{\ddot{a}}{a} \varphi+a^{2} \varphi+4 q \chi^{2} \varphi=0
$$

Now the background (i.e. the inflaton field) is not homogeneous anymore and the complete quantum problem is not easy. However, the efficiency of particle creation saves the day: the system rapidly becomes classical [86] and the problem can be handed over to a computer [56]-[58].

Instead of the number density of created particles it is more convenient (and rigorous) to measure the related quantity: the variance of the fluctuation field

$$
\left\langle\chi^{2}\right\rangle=\frac{1}{(2 \pi)^{3}} \int d^{3} k\left|\chi_{\vec{k}}\right|^{2}
$$

$\overline{2}$ For a discussion of the parametric resonance induced by an inflaton field see also refs. [84]. 
Initially fluctuations are small and the problem can be followed in the Hartree approximation. Namely, the field $\chi$ in Eq. (17) is replaced by its average, $\left\langle\chi^{2}\right\rangle$, and spatial gradients of the inflaton field are neglected. The formalism of section 3.1 then applies. In the situation of efficient particle creation occupation numbers grow and at some point quantum averages can be approximated by classical averages computed with the help of a certain distribution function $[87,86]$. At late times the problem becomes classical and it has to be supplemented with appropriate initial conditions which reflect early quantum evolution. Those are specified in the Fourier space as follows $[86,56]$. Amplitudes of mode functions are distributed with the probability density

$$
P\left[\chi_{\vec{k}}\right] \propto \exp \left[-\frac{2 \phi^{2}(0)}{m^{2}} \omega_{k}(0)\left|\chi_{\vec{k}}(0)\right|^{2}\right],
$$

and mode functions have random phases. The initial "velocities" are locked to their corresponding "coordinates"

$$
\dot{\chi}_{\vec{k}}(0)=-i \omega_{k}(0) \chi_{\vec{k}}
$$

The parameter $m^{2} / \phi^{2}(0)$ sets the scale of $\left\langle\chi^{2}\right\rangle$ which separates regions of quantum and classical fluctuations in this model. The semiclassical description is reliable as long as $g^{2} / 4 \pi=q m^{2} / 4 \pi \phi^{2}(0) \ll 1$.

Full non-linear problem can now be solved numerically on the lattice (namely, Eq. (17) and corresponding equation for the field $\chi(\tau, \mathbf{x})$ are solved directly in the configuration space). This classical problem accounts for all the effects of particle creation, their rescattering, inverse decays, etc. Initial stage of intensive growth of fluctuations (similar to parametric resonance, but not equivalent to it since the resonance parameter $q$ changes rapidly in the expanding Universe) is followed by the stage which is similar to the Kolmogorov turbulence when smooth power spectra of fluctuations are established which slowly approach equilibrium $[86,57,58,88]$.

An important quantity is the maximum strength of fluctuations achieved during the course of evolution (fluctuations are diluted by the expansion of the Universe after decay slows down). Compilation of results of Refs. [56,57] for the realistic case $m_{\phi}=10^{-6} M_{\mathrm{Pl}}$ is shown in Fig. 6 as a function of model parameters. The stars are results of the lattice calculation which takes all back reaction effects into account, solid lines correspond to the computationally less expensive Hartree approximation.

To compare this with the gravitational creation note that in the Hartree approximation $\rho_{X} / \rho \sim 2$ when fluctuations reach their maximum. Even the lowest level of fluctuations shown in Fig. 6 exceeds the amount of gravitation- 


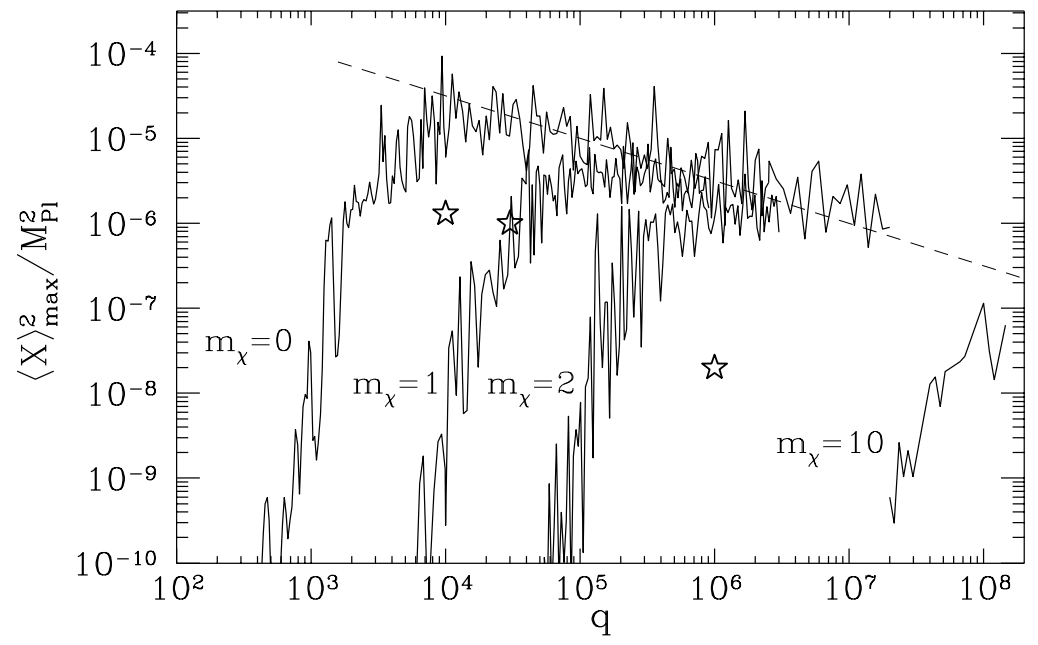

Fig. 6. The maximum value of the variance $\left\langle X_{\max }^{2}\right\rangle$ as a function of $q$. Solid lines correspond to the Hartree approximation with different values of $m_{\chi} \equiv m_{X} / m_{\phi}$, Ref. [56]. The stars are results of fully non-linear lattice calculations with $m_{X} \ll m_{\phi}$, Ref. [57].

ally created particles. However, while stable particles will be generally overproduced, this mechanism still can be relevant for dark matter and UHECR phenomenon, when e.g. for a given value of $m_{X}$ the value of $q$ is low enough to prevent effective particle creation, but not negligible. Fig. 6 shows that particles 10 times heavier than inflaton are produced and even this is not a limit in the case of very large $q[89]$.

In the opposite situation when $q$ is sufficiently large and fluctuations develop to the full strength, an interesting and important phenomenon of non-thermal phase transitions [16] can occur which is the subject of discussion in the following section.

\section{Topological defects and inflation}

Decaying topological defect can naturally produce very energetic particles, and this may be related to UHECR [41-46], for recent reviews see [26]. However, among motivations for inflation there was the necessity to get rid of unwanted topological defects. And inflation is doing this job excellently. Since temperature after reheating is constrained, especially severely in supergravity models, it might be that the Universe was never reheated up to the point of GUT phase transitions. Topological defects with a sufficiently high scale of symmetry breaking cannot be created. Then, how could such topological defects populate the Universe? 
The answer may be provided by non-thermal phase transitions [16] which can occur in preheating after inflation. Explosive particle production caused by stimulated decay of inflaton oscillations lead to anomalously high field variances which restore symmetries of the theory even if the actual reheating temperature is small. The defects are formed when variances are reduced by the continuing expansion of the Universe and a phase transition occurs.

\subsection{Non-thermal phase transitions}

Let us first describe ideas qualitatively. Let the inflaton oscillations which have amplitude $\phi(0) \sim M_{\mathrm{Pl}}$ decay rapidly into some field $X$. In the instant process of decay the energy conserves and we have $m_{\phi}^{2} M_{\mathrm{Pl}}^{2} \sim k^{2}\left\langle X^{2}\right\rangle$, where on the left hand side of this equality we write the initial inflaton energy and on the right hand side we write the final energy stored in the X-field. The inflaton decays much faster than the system thermalizes, therefore typical momentum of $X$ particles is of order of the inflaton mass, $k \sim m_{\phi}$. We find $\left\langle X^{2}\right\rangle \sim M_{\mathrm{Pl}}^{2}$. In thermal equilibrium with the temperature $T$ we would have $\left\langle X^{2}\right\rangle=T^{2} / 12$. In this respect the strength of fluctuations is the same as it would be in equilibrium with the Plankian temperature despite the fact that the real reheating temperature is much lower. Of course, this is an extreme estimate, mainly because the expansion of the Universe was neglected. The realistic $\left\langle X^{2}\right\rangle$ is shown in Fig. 6, but it can still exceed equilibrium temperature by many orders of magnitude.

This effect is important for the behaviour of spontaneously broken symmetries at preheating. Let us consider the model in which in the vacuum state the symmetry is broken by an order parameter $\Phi$. At the tree level this can be described by the potential

$$
V(\Phi)=-\mu^{2} \Phi^{2} / 2+\lambda_{\Phi} \Phi^{4} / 4
$$

The parameter $\mu$ is related to the symmetry breaking scale via $\mu^{2}=\lambda_{\Phi} \Phi_{0}^{2}$. If the filed $\Phi$ and the product of inflaton decay, $X$, are coupled (with corresponding interaction term in the Lagrangian being $\left.\alpha X^{2} \Phi^{2} / 2\right)$, at non-zero density

of X-particles the effective mass of $\Phi$ field changes to $m_{\text {eff }}^{2}=-\mu^{2}+\alpha\left\langle X^{2}\right\rangle$. The symmetry is restored if the effective mass became positive, $\left\langle X^{2}\right\rangle>\lambda_{\Phi} \Phi_{0}^{2} / \alpha$.

The real problem is complicated and model dependent. While some features can be anticipated and some quantities roughly estimated, the issue requires numerical studies. In recent papers [90-92] the defect formation and even the possibility of the first order phase transitions during preheating was demonstrated explicitly. 


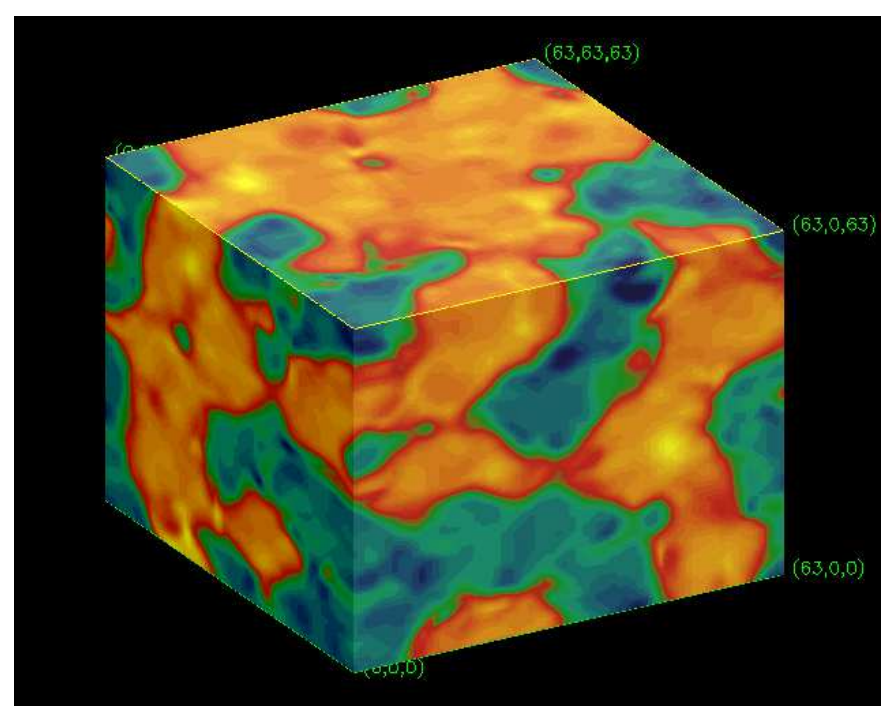

Fig. 7. Domain wall structure generated in simulation of preheating in the model Eq. (22) with $M=1, g=0$, Ref. [93].

\subsection{Topological defects in simple models}

Let us consider for simplicity the system when one and the same field serves as the inflaton and the symmetry breaking parameter, $\Phi \equiv \phi$. We derive the set of models from the prototype potential

$$
V(\phi)=\frac{\lambda}{4}\left(\phi^{2}-v^{2}\right)^{2}-\frac{g^{2}}{2} \phi^{2} X^{2} .
$$

The inflaton scalar field $\phi$ has $M$ components, $\phi^{2}=\sum_{i=1}^{M} \phi_{i}^{2}$, and interacts with an $N$-component scalar field $X, X^{2}=\sum_{i=1}^{N} X_{i}^{2}$. For simplicity, the field $X$ is taken massless and without self-interaction. The fields have minimal coupling to gravity in a FRW universe with a scale factor $a(t)$. It is assumed that the inflaton oscillations start along $\phi_{1}$ direction. In the effective mass of $\phi$ there are contributions from $g^{2}\left\langle X^{2}\right\rangle$ as well as $\propto \lambda\left\langle(\delta \phi)^{2}\right\rangle$.

We review several models, in the order of increasing complexity. Initial conditions in all cases correspond to the vacuum for fluctuations. System then evolved through particle creation, their rescattering, phase transitions and finally defects creation.

\subsubsection{Domain walls [93]}

Consider one component inflaton field, $M=1$, and no $X$-fields. Relevant topological structures in the double-well potential are domain walls. Even in the model without fields $X$, fluctuations of the field $\phi$ grow dramatically 


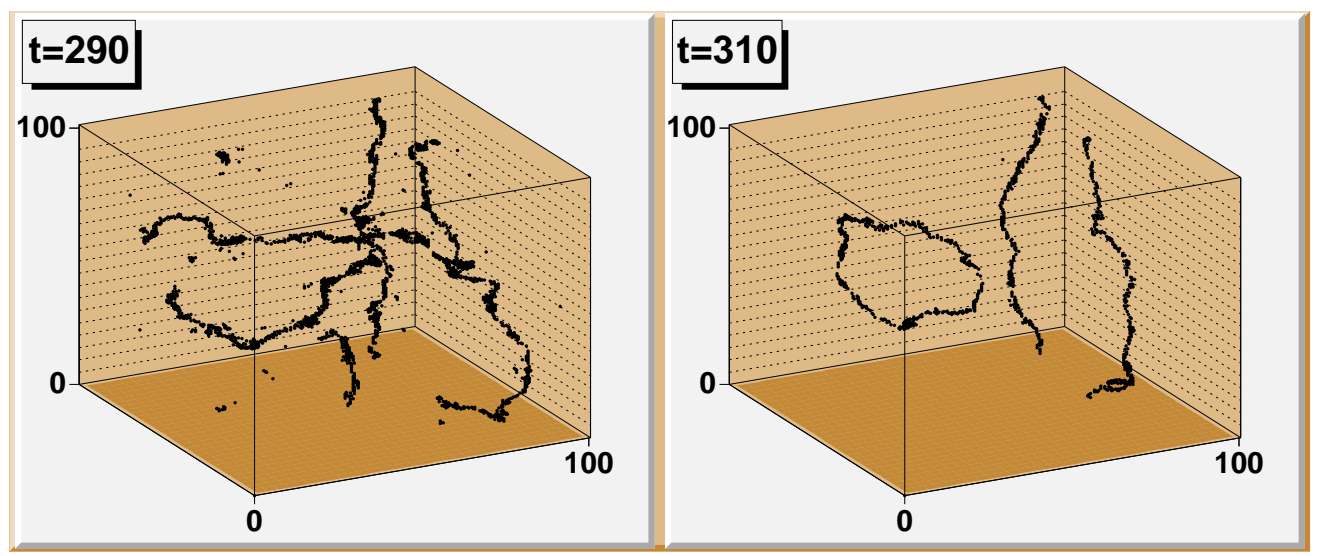

Fig. 8. String network generated in simulation of preheating in the model Eq. (22) with $M=2, g=0$ (see Ref. [91]) is shown at two successive moments of time.

(because of the self-interaction $\propto \lambda$, the zero mode can decay in the process $2 \phi \rightarrow 4 \phi$; in addition to that the spinodal decomposition is effective in the present model). This leads to formation of a domain structure at certain values of $\lambda$. The domain structure which emerged in such situation is shown in Fig. 7

\subsubsection{Strings [91]}

Again, consider the model without $X$-fields, but the inflaton has two components, $M=2$. Relevant topological structures in the Mexican hat potential are strings.

Fluctuations of the fields $\phi_{1}$ and $\phi_{2}$ during preheating restore symmetry along $\phi_{1}$ direction, but along $\phi_{2}$ direction symmetry is broken. For some period of time the universe is divided into domains filled with the field $\phi_{2} \approx \pm \mathrm{v}$. Gradually the amplitude of fluctuations of the fields $\phi_{i}$ decreases, and symmetry $\phi_{1} \rightarrow-\phi_{1}$ also breaks down. At this moment the seed domain structure is transformed into a string network. Fig. 8 shows the string distribution in a simulation with the symmetry breaking scale $\mathrm{v}=3 \times 10^{16} \mathrm{GeV}$, when a pair of "infinite" strings and one big loop were formed. Size of the box is comparable to the Hubble length at this time.

The final result is the string formation, but the sequence of symmetry breaking patterns would be unusual for thermal phase transitions. For example, the $\mathrm{O}(2)$ symmetry was only partially restored. Another peculiarity of non-thermal phase transitions is the possible presence of oscillating zero mode during final stages of the phase transition. This makes probability of formation of long strings (as well as domains in the previous model) to be a non-monotonic function of $v$, at least for large $v$ and in simplest models considered so far. In more complicated models inflaton oscillations can decay completely before the phase transition, and the phase transition can be even of the first order. 
Defect formation in such models will be more robust and resembling more closely phase transitions in thermal background.

\subsubsection{First order phase transition [90]}

Here we consider one component inflaton field, $M=1$ and several $X$-fields. In the case $g^{2} / \lambda \gg 1$ the phase transition can be of the first order. This can be expected by analogy with the usual thermal case [94]. The necessary conditions for this transition to occur and to be of the first order are as follows.

(i) At the moment of phase transition a local minimum of the effective potential should be at $\phi=0$, which gives $g^{2}\left\langle X^{2}\right\rangle>\lambda v^{2}$.

(ii) At the same time, the typical momentum $p_{*}$ of $X$ particles should be smaller than $g v$. This is the condition of the existence of a potential barrier. Particles with momenta $p<g v$ cannot penetrate the state with $|\phi| \approx v$, so they cannot change the shape of the effective potential at $|\phi| \approx v$. Therefore, if both conditions (i) and (ii) are satisfied, the effective potential has a local minimum at $\phi=0$ and two degenerate minima at $\phi \approx \pm v$.

(iii) Before the minima at $\phi \approx \pm v$ become deeper than the minimum at $\phi=0$, the inflaton's zero mode should decay significantly, so that it performs small oscillations near $\phi=0$. Then, after the minimum at $|\phi| \approx v$ becomes deeper than the minimum at $\phi=0$, fluctuations of $\phi$ drive the system over the potential barrier, creating an expanding bubble.

All these conditions can be met more easily at large $g^{2} / \lambda \gg 1$ and with several $X$-fields. Results discussed below were obtained [90] on a $128^{3}$ lattice in the model with parameters $g^{2} / \lambda=200$ and $v=0.7 \times 10^{-3} M_{\mathrm{Pl}} \approx 0.8 \times 10^{16} \mathrm{GeV}$, for a two-component $X$, with the expansion of the universe assumed to be radiation dominated (similar behaviour was observed in the model with single $X$ field, i.e. $N=1$, as well).

Time dependence of the zero mode $\phi_{0}$ is shown in Fig. 9. Initially $\phi_{0}$ oscillates with a large amplitude $\bar{\phi} \gg v$. If all fluctuations were absent, the zero mode $\phi_{0}$, because of its dilution in the expanding universe, would at some time be unable to cross the potential barrier at $v=0$ and would start oscillations near one of its vacuum values, $\pm v$. This would happen when the amplitude of the oscillations becomes smaller than $\sqrt{2} v$. In Fig. 9 we see that the actual dynamics is completely different. The zero mode of the field $\phi$ continues to oscillate near $\phi=0$ even when its amplitude becomes much smaller than $v$. In other words, the field oscillates on top of the local maximum of the bare potential. This can occur only because the effective potential acquires a minimum at $\phi=0$ due to interaction of the field $\phi$ with $\left\langle X^{2}\right\rangle$. And amplitude

of oscillations is decreasing much faster than it would decrease due to the 


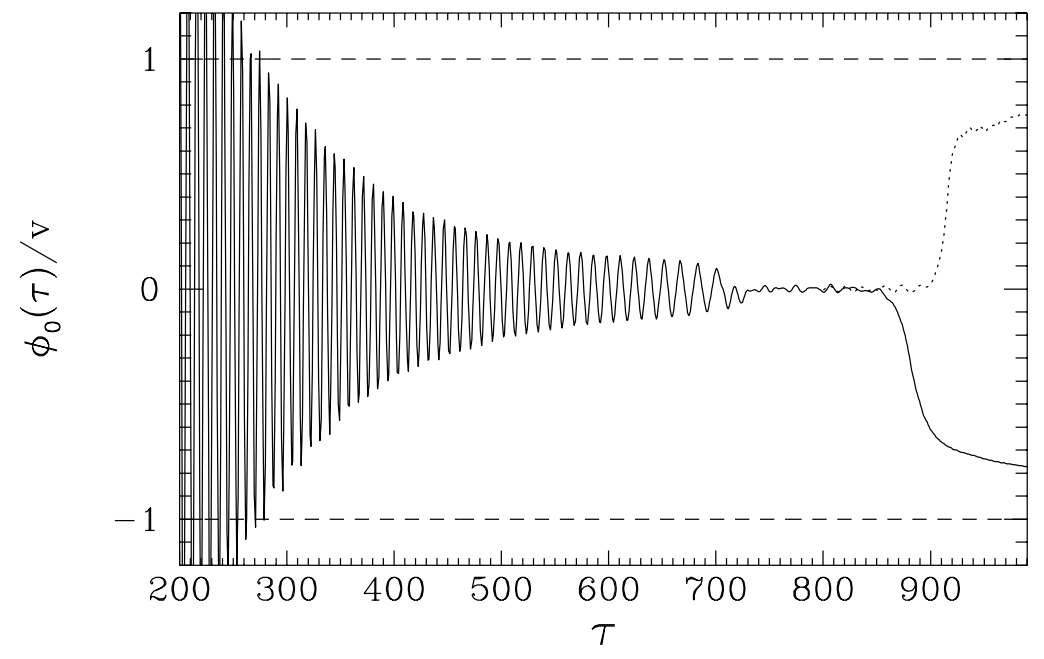

Fig. 9. Time dependence of the zero-momentum mode of $\phi$ in units of its vacuum value for two runs with different realizations of random initial conditions for fluctuations in simulation of preheating in the model Eq. (22) with $M=1, g \neq 0$, Ref. $[90]$.

simple expansion of the Universe. This happens because of continuing decay of the zero mode into $X$-particles.

At still later times, $\tau \gtrsim 720$ in Fig. 9, the zero mode of $\phi$ decays completely. This should be interpreted as restoration of the symmetry $\phi \rightarrow-\phi$ by nonthermal fluctuations. Finally, at $\tau \gtrsim 860$ (when $X$-fluctuations were diluted sufficiently by the expansion) a phase transition occurs and the symmetry breaks down. In runs with different realizations for initial ("vacuum") fluctuations the system ends up either in $+v$ or in $-v$ vacuum and the transition happens at different time moments. It was shown that transition is triggered in this model by a spontaneous nucleation of a bubble of the new phase and the bubble's subsequent expansion until it was occupying the whole integration volume. The field configuration at the beginning of the phase transition is shown in Fig. 10, where the surface of the constant field $\phi=-0.7 v$ is plotted at the beginning of the phase transition. Inside the surface $\phi<-0.7 v$. (Minimum of the effective potential is shifted somewhat from the vacuum value at this time, see Fig. 9, because the contribution of fluctuations is still significant).

Models that exhibit behaviour shown in Fig. 9 will lead to the domain structure surviving until present. This would be a cosmological disaster and such a class of models is ruled out [1].

However, different behaviour of the zero mode is observed at smaller values of $g^{2} / \lambda$ or $N$ (but still $g^{2} / \lambda \gg 1$ ). There, the phase transition can occur when the zero mode still oscillates near $\phi=0$ with a relatively large amplitude. This 


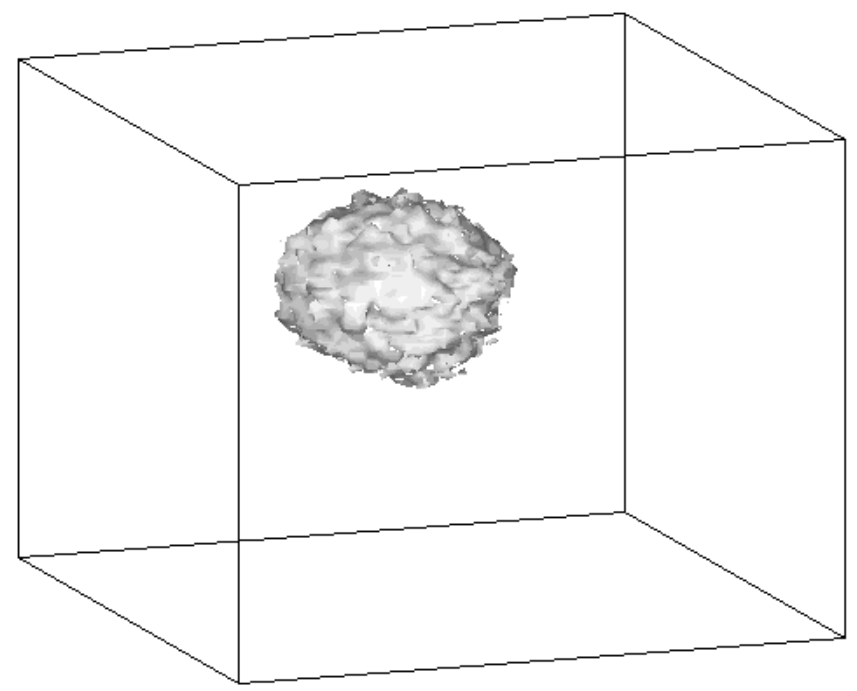

Fig. 10. Spontaneously nucleated bubble of the new phase at the initial stage of its expansion, Ref. [90].

is a new, specifically nonthermal, type of a phase transition. In such cases, bubbles of $+v$ and $-v$ phases will be nucleated in turn (most often at the maximum of amplitude of the zero mode when it is in the closest proximity to the top of the potential barrier), but their abundances need not be equal, and for certain values of the parameters one of the phases may happen not to form infinite domains. Such models are not ruled out and in fact may have interesting observable consequences, e.g. an enhanced (by bubble wall collisions) background of relic gravitational waves produced by the mechanism proposed in Ref. [18].

There are no reasons to doubt that in more complicated models non-thermal phase transitions may lead to creation of magnetic monopoles, or magnetic monopoles connected by strings (necklaces [43]), the latter being the most favourable topological defect candidate for explanation of UHECR [49].

\section{Conclusions}

Next generation cosmic ray experiments, like the Pierre Auger Project [95], the High Resolution Fly's Eye [96], the Japanese Telescope Array Project [97], and the Orbiting Wide-angle Light-collector (OWL) [98], will tell us which model for UHECR may be correct and which has to be ruled out.

Very weakly interacting superheavy X-particles with $m_{X}=($ a few $) \cdot 10^{13} \mathrm{GeV}$ may naturally constitute a considerable fraction of Cold Dark Matter. These particles are produced in the early Universe from vacuum fluctuations dur- 
ing or after inflation. Decays of X-particles may explain UHE cosmic rays phenomenon. Related density fluctuations may have left an imprint in fluctuations of cosmic microwave background radiation if scalar X-particles with minimal coupling to gravity are approximately twice heavier than the inflaton and $\Omega_{X} \sim 1$.

If UHE cosmic rays are indeed due to the decay of these superheavy particles, there has to be a new sharp cut-off in the cosmic ray spectrum at energies somewhat smaller than $m_{X}$. Since the number density $n_{X}$ depends exponentially upon $m_{X} / m_{\phi}$, the position of this cut-off is fixed and can be predicted to be near $m_{\phi} \approx 10^{13} \mathrm{GeV}$, the very shape of the cosmic ray spectrum beyond the GZK cut-off being of quite generic form following from the QCD quark/gluon fragmentation.

Very discriminating signature is related to anisotropy of cosmic rays. If particles immune to CMBR are there, the UHECR events should point towards distant (i.e. beyond GZK sphere), extraordinary astrophysical sources [99]. If superheavy relic particles are in the game, the Galaxy halo will be reflected in anisotropy of the UHECR flux [100-102]. If neither will be true but UHECR will point instead to "local" galaxies with evidence for a central supermassive black hole, that would imply that the existence of a black hole dynamo is not a sufficient condition for the presence of pronounced jets and UHECRs are created by the remnants of dead quasars [32]. If none of the above will be true, arrival directions will be almost isotropic on large angular scales but UHECRs will cluster on small scales, then perhaps extragalactic magnetic fields are much stronger than previously thought [103], or perhaps cosmic necklaces do exist.

It is remarkable that we might be able to learn about the earliest stages of the Universe's evolution by studying UHECRs. Discovery of heavy relic Xparticles will mean that the model of inflation is likely correct, or that at least standard early Friedmann evolution from the singularity is ruled out, since otherwise X-particles would have been inevitably overproduced $[19,104]$.

We conclude that the observations of Ultra High Energy cosmic rays can probe the spectrum of elementary particles in the superheavy range and can give an unique opportunity for investigation of the earliest epoch of evolution of the Universe, starting with the amplification of vacuum fluctuations during inflation through fine details of gravitational interaction and down to the physics of reheating. 


\section{Acknowledgements}

The work of V.K. was supported partially by the Russian Fund for Fundamental Research grant 98-02-17493-a.

\section{References}

[1] Ya. B. Zeldovich, I. Yu. Kobzarev and L. B. Okun. Zh. Eksp. Teor. Fiz. 67 (1974) 3 [Sov. Phys. JETP 40 (1974) 1 ].

[2] Ya. B. Zeldovich and M. Yu. Khlopov, Phys. Lett. 79B (1978) 239.

[3] D. A. Kirzhnits, JETP Lett. 15 (1972) 529; D. A. Kirzhnits and A. D. Linde, Phys. Lett. 42B (1972) 471; Sov. Phys. JETP 40 (1974) 628; Ann. Phys. 101 (1976) 195; S. Weinberg, Phys. Rev. D9 (1974) 3320; L. Dolan and R. Jackiw, Phys. Rev. D9 (1974) 3357.

[4] Yu. Kobzarev, L. B. Okun, and M. B. Voloshin, Yad. Fiz. 20 (1974) 1229 [Sov. J. Nucl. Phys. 20 (1975) 644; S. Coleman, Phys. Rev. D15 (1977) 2922; S. Coleman and F. DeLuccia, Phys. Rev. D21 (1980) 3305; V. A. Berezin, V. A. Kuzmin, and I. I. Tkachev, Phys. Lett. 120B (1983) 91; J. Ipser and P. Sikivie, Phys. Rev. D30 (1984) 712; V. A. Berezin, V. A. Kuzmin, and I. I. Tkachev, Phys. Rev. D36 (1987) 2919; S. K. Blau, E. I. Guendelman, and A. H. Guth, Phys. Rev. D35 (1987) 1747.

[5] A. A. Starobinskii, Phys. Lett. 91B (1980) 99.

[6] A. H. Guth, Phys. Rev. D23 (1981) 347-356.

[7] A. D. Linde, Phys. Lett. 108B (1982) 389; A. Albrecht and P. J. Steinhardt, Phys. Rev. Lett. 48 (1982) 1220.

[8] A. D. Linde, Phys. Lett. 129B (1983) 177.

[9] A. D. Linde, Particle Physics and Inflationary Cosmology (Harwood, Chur, Switzerland, 1990).

[10] E. W. Kolb and M. S. Turner, The Early Universe, (Addison-Wesley, Reading, Ma., 1990).

[11] V. F. Mukhanov and G. V. Chibisov, JETP Lett. 33 (1981) 532; S. W. Hawking, Phys. Lett. 115B (1982) 295 ; A. A. Starobinsky, Phys. Lett. 117B (1982) 175; A. H. Guth and S.-Y. Pi, Phys. Rev. Lett. 49 (1982) 1110 .

[12] G.F. Smoot et al., Ap. J. 396 (1992) L1; http://www.gsfc.nasa.gov/astro/cobe/cobe_home.html.

[13] http://map.gsfc.nasa.gov/. 
[14] http://astro.estec.esa.nl/Planck/.

[15] http://www.sdss.org/.

[16] L. Kofman, A. Linde, and A. A. Starobinsky, Phys. Rev. Lett. 76 (1996) 1011; I. I. Tkachev, Phys. Lett. B376 (1996) 35.

[17] E. W. Kolb, A. D. Linde and A. Riotto, Phys. Rev. Lett. 77 (1996) 4290;

E. W. Kolb, A. Riotto and I. I. Tkachev, Phys. Lett. B423 (1998) 348.

[18] S. Y. Khlebnikov and I. I. Tkachev, Phys. Rev. D56 (1997) 653.

[19] V. A. Kuzmin and I. I. Tkachev, JETP Lett. 68 (1998) 271.

[20] K. Greisen, Phys. Rev. Lett. 16 (1966) 748;

G. T. Zatsepin and V. A. Kuzmin, Pisma Zh. Eksp. Teor. Fiz. 4 (1966) 114.

[21] N. N. Efimov et al., Proc. International Symposium on Astrophysicsl aspects of the most energetic cosmic rays, eds. M. Nagano and F. Takahara (1991, World Scientific, Singapore), p. 20; T. A. Egorov et. al., in: Proc. Tokyo Workshop on Techniques for the Study of Extremely High Energy Cosmic Rays, ed. M.Nagano (ICRR, U. of Tokyo, 1993); B. N. Afanasiev, Proc. International Symposium on Extremely High Energy Cosmic Rays: Astrophysics and future observations, ed. M. Nagano (Institute for Cosmic Rays Research, Tokyo, 1996).

[22] M. A. Lawrence, R. J. O. Reid, and A. A. Watson, J. Phys. G Nucl. Part. Phys. 17 (1991) 733; http://ast.leeds.ac.uk/haverah/hav-home.html.

[23] D. J. Bird et. al., Phys. Rev. Lett. 71 (1993) 3401; Astroph. J. 424 (1994) 491; 441 (1995) 144.

[24] N. Hayashida et. al., Phys. Rev. Lett. 73 (1994) 3491; http://icrsun.icrr.utokyo.ac.jp/as/project/agasa.html.

[25] V. S. Berezinsky, S. V. Bulanov, V. A. Dogiel, V. L Ginzburg, and V. S. Ptuskin, "Astrophysics of cosmoc Rays", Elsevier, 1990.

[26] P. Bhattacharjee and G. Sigl, astro-ph/9811011; V. Berezinsky, astro$\mathrm{ph} / 9811268$.

[27] Takeda M. et al., Phys. Rev. Lett. 81 (1998) 1163.

[28] M. A. Ruderman and Sutherland, Ap. J. 196 (1975) 51; R. D. Blandford and R. Znajek, MNRAS 179 (1977) 433; A. M. Hillas, Ann. Rev. Astron. Astrophys. 22 (1984) 425.

[29] C. A. Norman, D. B. Melrose, and A. Achterberg, Ap. J. 454 (1995) 60.

[30] J. W. Elbert and P. Sommers, Ap. J. 441 (1995) 151.

[31] A. Dar, astro-ph/9901005.

[32] E. Boldt and P. Ghosh, astro-ph/9902342. 
[33] S. Coleman and S. L. Glashow, hep-ph/9808446.

[34] G. R. Farrar, Phys. Rev. Lett. 76 (1996) 4111; D. J. Chung, G. R. Farrar, and E. W. Kolb, Phys. Rev. D57 (1998) 4696.

[35] S. Raby, Phys. Lett. B422 (1998) 158.

[36] M. B. Voloshin and L. B. Okun, Sov. J. Nucl. Phys. 43 (1986) 495.

[37] T. J. Weiler, hep-ph/9710431; D. Fargion, B. Mele, and A. Salis, astroph/9710029; S. Yoshida, G. Sigl, and S. Lee, Phys. Rev. Lett. 58 (1998) 55.

[38] G. Gelmini and A. Kusenko, hep-ph/9902354.

[39] J. Bahcall and E. Waxman, hep-ph/9902383.

[40] W. H. Kinney and A. Riotto, hep-ph/9903459; J. Adams and S. Sarkar, talk presented at the ICTP Workshop on physics of relic neutrinos, Trieste, Italy, 1998.

[41] C. T. Hill, D. N. Schramm and T. P. Walker, Phys. Rev. D36 (1987) 1007.

[42] J. H. MacGibbon and R. H. Brandenberger, Nucl. Phys. B331 (1990) 153; P. Bhattacharjee and N. C. Rana, Phys. Lett. B246 (1990) 356.

[43] V. Berezinsky, X. Martin and A. Vilenkin, Phys. Rev. D56 (1997) 2024; V. Berezinsky and A. Vilenkin Phys. Rev. Lett. 79 (1997) 5202.

[44] R. L. Davis and E. P. S. Shellard, Phys. Lett. B209 (1988) 485; R. Brandenberger et al, Phys. Rev. D54 (1996) 6059; C.J.A.P. Martins and E. P. S. Shellard, hep-ph/9806480.

[45] C. T. Hill, Nucl. Phys. B224 (1983) 469; P. Bhattacharjee and G. Sigl, Phys. Rev. D51 (1995) 4079.

[46] T. W. Kephart and T. J. Weiler, Astropart. Phys. 4 (1996) 271.

[47] V. A. Kuzmin and V. A. Rubakov, Talk at the Workshop "Beyond the Desert", Castle Rindberg 1997; Phys. Atom. Nucl. 61 (1998) 1028 [Yad. Fis. 61 (1998) $1122]$.

[48] V. Berezinsky, M. Kachelriess and A. Vilenkin, Phys. Rev. Lett. 79 (1997) 4302 ;

[49] V. Berezinsky, P. Blasi, and A. Vilenkin, Phys. Rev. D58 (1998) 103515.

[50] M. Birkel and S. Sarkar, Astropart. Phys. 9 (1998) 297.

[51] K. Benakli, J. Ellis, and D. V. Nanopoulos, Phys. Rev. D59 (1999) 047301; K. Benakli, Phys. Lett. B447 (1999) 51; D. V. Nanopoulos, hep-ph/9809546.

[52] T. Han, T. Yanagida and R.J. Zhang, Phys. Rev. D58 (1998) 095011.

[53] K. Hamaguchi, Y. Nomura, and T. Yanagida, Phys. Rev. D58 (1998) 103503; Phys. Rev. D59 (1999) 063507. 
[54] M. Kachelriess, hep-ph/9806322. R. Dick, N. Eschrich and M. Gaul, hepph/9808245; G. K. Leontaris and J. Rizos, hep-ph/9901098; K. Hamaguchi, K. I. Izawa and Y. Nomura, hep-ph/9903207.

[55] L. Kofman, A. Linde, and A. Starobinsky, Phys. Rev. Lett. 73 (1994) 3195;

[56] S. Y. Khlebnikov and I. I. Tkachev, Phys. Lett. B390 (1997) 80.

[57] S. Y. Khlebnikov and I. I. Tkachev, Phys. Rev. Lett. 79 (1997) 1607.

[58] T. Prokopec and T. G. Roos, Phys. Rev. D55 (1997) 3768; B. R. Greene, T. Prokopec, and T. G. Roos, Phys. Rev. D 56 (1997) 6484.

[59] D. J. H. Chung, E. W. Kolb and A. Riotto, Phys. Rev. D59 (1999) 023501.

[60] V. A. Kuzmin and I. I. Tkachev, hep-ph/9809547.

[61] J. Ellis, J. Kim and D. V. Nanopoulos, Phys. Lett. B145 (1984) 181.

[62] E. W. Kolb, D. J. H. Chung, and A. Riotto, Phys. Rev. Lett. 81 (1998) 4048; hep-ph/9809453.

[63] S. G. Mamaev, V. M. Mostepanenko, and A. A. Starobinskii, ZhETF 70 (1976) 1577 [ Sov. Phys. JETP 43 (1976) 823].

[64] S. G. Mamaev, V. M. Mostepanenko, and V. M. Frolov, Yad. Fiz. 23 (1996) 1118, ibid 26 (1997) 215.

[65] L. Parker, Phys. Rev. 183 (1969) 1057; A. A. Grib and S. G. Mamaev, Yad. Fiz. 10 (1969) 1276 [Sov. J. Nucl. Phys. 10 (1970) 722 ]; Ya. B. Zel'dovich and A. A. Starobinslii, Zh. Exp. Theor. Fiz. 61 (1971) 2161 [Sov. Phys. JETP 34 (1972) 1159]; L. P. Grishchuk, Sov. Phys. JETP 40 (1975) 409.

[66] A. A. Grib, S. G. Mamaev, and V. M. Mostepanenko, Quantum Effects in Strong External Fields, [in Russian], Atomic Energy Press, Moscow (1980).

[67] N. N. Bogolubov, Sov. Phys. JETP 7 (1958) 51.

[68] N. A. Chernikov and E. A. Tagirov, Ann. Inst. Henri Poinare 9A, 109 (1968); E. A. Tagirov, Ann. Phys. 76 (1973) 561; T. S. Bunch and P. C. W. Davies, Proc. R. Soc. A360 (1978) 117.

[69] A. D. Linde, Phys. Lett. 116B (1982) 335; A. A. Starobinsky, Phys. Lett. 117B (1982) 175; A. Vilenkin and L. H. Ford, Phys. Rev. D26 (1982) 1231; B. Allen, Phys. Rev. D32 (1985) 3136; C. M. Massacand and C. Schmid, Ann. Phys. 231 (1994) 363; H. F. Muller and C. Schmid, gr-qc/9412021; gr-qc/9412022.

[70] A. A. Starobinsky and J. Yokoyama, Phys. Rev. D50 (1994) 6357.

[71] D. Lyth and D. Roberts, hep-ph/9609441.

[72] K. Enqvist, K. W. Ng and K. A. Olive, Nucl. Phys. B303 (1988) 713. 
[73] A. A. Starobinsky and V. Sahni, in: Proc. 6th Soviet Gravitation Conf., ed. V. N. Ponomarev (MGPI Press, Moscow, 1984); G. Efstathiou and J. R. Bond, Mon. Not. R. Astron. Soc. 218 (1986) 103; H. Kodama and M. Sasaki, Int. J. Mod. Phys. A2 (1987) 491; A. A. Starobinsky, Sov. Astron. Lett. 14 (1988) 166.

[74] A. Linde and V. Mukhanov, Phys. Rev. D56 (1997) 535.

[75] P. J. E. Peebles, astro-ph/9805194; astro-ph/9805212.

[76] L. H. Ford, Phys. Rev. D35 (1987) 2955; M. Joyce and T. Prokopec, Phys. Rev. D57 (1998) 6022; P. J. E. Peebles and A. Vilenkin, astro-ph/9810509; G. Felder, L. Kofman, and A. Linde, hep-ph/9903350.

[77] J. Preskill, M. B. Wise, and F. Wilczek, Phys. Lett. 120B (1983) 127; L. F. Abbott and P. Sikivie, Phys. Lett. 120B (1983) 133.

[78] J. Ellis, D. V. Nanopoulos and M. Quiros, Phys. Lett. B174 (1986) 176.

[79] I. I. Tkachev, Sov. Astron. Lett. 12 (1986) 305; I. I. Tkachev, Phys. Lett. B191 (1987) 41; I. I. Tkachev, Phys. Lett. B261 (1991) 289.

[80] J. Garcia-Bellido and A. Kusenko, hep-ph/9812534.

[81] T. W. Kephart and T. J. Weiler, Phys. Rev. D52 (1995) 3226.

[82] C. J. Hogan and M. J. Rees, Phys. Lett. B205 (1988) 228; E. Kolb and I. I. Tkachev, Phys. Rev. Lett. 71 (1993) 3051; Phys. Rev. D 49 (1994) 5040; Phys. Rev. D50 (1994) 769; Ap. J. 460 (1996) L25.

[83] A. D. Dolgov and A. D. Linde, Phys. Lett. B116 (1982) 329; L. F. Abbott, E. Farhi, and M. B. Wise, Phys. Lett. B117 (1982) 29; A. Albrecht, P. J. Steinhardt, M. S. Turner, and F. Wilczek, Phys. Rev. Lett. 48 (1982) 1437; A. D. Dolgov and D. P. Kirilova, Sov. Nucl. Phys. 51 (1990) 172;

[84] J. Traschen and R. Brandenberger, Phys. Rev. D 42 (1990) 2491; Y. Shtanov, J. Traschen, and R. Brandenberger, Phys. Rev. D 51 (1995) 5438; D. Boyanovsky, H. J. de Vega, R. Holman, D. S. Lee, and A. Singh, Phys. Rev. D 51 (1995) 4419; M. Yoshimura, Prog. Theor. Phys. 94 (1995) 8873; D. Kaiser, Phys. Rev. D 53 (1996) 1776.

[85] L. Kofman, A. Linde, and A. A. Starobinsky, Phys. Rev. D 56 (1997) 3258.

[86] S. Y. Khlebnikov and I. I. Tkachev, Phys. Rev. Lett. 77 (1996) 219.

[87] D. Polarski and A. A. Starobinsky, Class. Quant. Grav. 13 (1996) 377.

[88] D. T. Son, Phys. Rev. D54 (1996) 3745.

[89] D.J.H. Chung, hep-ph/9809489; G. Felder, L. A. Kofman, and A. D. Linde, hep-ph/9812289.

[90] S. Khlebnikov, L. Kofman, A. Linde, and I. Tkachev, Phys. Rev. Lett. 81 (1998) 2012. 
[91] I. Tkachev, S. Khlebnikov, L. Kofman, and A. Linde, Phys. Lett. B440 (1998) 262.

[92] S. Kasuya and M. Kawasaki, Phys. Rev. D58 (1998) 083516.

[93] S. Khlebnikov, L. Kofman, A. Linde, and I. Tkachev, unpublished; I. Tkachev, talk at 18th Texas Symposium on Relativistic Astrophysics, Chicago, IL, 15-20 Dec 1996, hep-ph/9701376.

[94] D.A. Kirzhnits and A.D. Linde, Ann. Phys. (N.Y.) 101 (1976) 195.

[95] J. W. Cronin, Nucl. Phys. B (Proc. Suppl.) 28 (1992) 213; The Pierre Auger Observatory Design Report (2nd edition), March 1997; M. Boratav, for Pierre Auger Collaboration, The Pierre Auger Observatory Project: An Overview., Proc. of 25th International Cosmic Ray Conference, Durban, v. 5, p. 205 (1997); http://www.auger.org.

[96] S. C. Corbato et al., Nucl. Phys. B (Proc. Suppl.) 28 (1992) 36; http://bragg.physics.adelaide.edu.au/astrophysics/HiRes.html.

[97] M. Teshima et al., Nucl. Phys. B (Proc. Suppl.) 28 (1992) 169; http://www-ta.icrr.u-tokyo.ac.jp.

[98] J. F. Ormes et al., Proc. 25th International Cosmic Ray Conference (Durban, 1977), eds: M. S. Potgieter et al., Vol. 5, 273; http://lheawww.gsfc.nasa.gov/docs/gamcosray/hecr/OWL/.

[99] G. R. Farrar and P. L. Biermann, Phys. Rev. Lett. 81 (1998) 3579.

[100] S. L. Dubovskii and P. G. Tinyakov JETP Lett. 68 (1998) 107.

[101] V. Berezinskii and A. A. Mikhailov, astro-ph/9810277.

[102] M. Takeda et al, astro-ph/9902239.

[103] M. Lemoine, G. Sigl, and P. Biermann, astro-ph/9903124; D. Ryu and P. L. Biermann, Astron. Astrophys. 335 (1998) 19.

[104] I. I. Tkachev, talk at the conference on Particle Physics and the Early Universe (COSMO 98), Monterey, CA, 15-20 Nov 1998; hep-ph/9901428. 